\title{
3. Öffentliche Entwicklungshilfe
}

\section{Gérard Perroulaz and Antonio Girardi}

\section{(2) OpenEdition}

\section{Journals}

Electronic version

URL: http://journals.openedition.org/sjep/287

ISSN: 1663-9677

Publisher

Institut de hautes études internationales et du développement

Printed version

Date of publication: 1 avril 2003

Number of pages: $279-296$

ISBN: 2-88247-050-3

ISSN: 1660-5926

\section{Electronic reference}

Gérard Perroulaz und Antonio Girardi, « 3. Öffentliche Entwicklungshilfe », Schweizerisches Jahrbuch für Entwicklungspolitik [Online], 22-1 | 2003, Online erschienen am: 07 Mai 2010, abgerufen am 07 September 2020. URL : http://journals.openedition.org/sjep/287 


\section{3. ÖFFENTLLCHE ENTWICKLUNGSHILFE}

KOMMENTAR

Entwicklung der öffentlichen Hilfe der Schweiz und Vergleich mit sämtlichen DAC-Ländern (Grafiken 7 bis 10)

Der OECD-Entwicklungshilfeausschuss (DAC) gibt statistische Angaben über die für die Entwicklungsländer bestimmten finanziellen Mittel heraus. Dabei unterscheidet das DAC die (im statistischen Aggregat als „öffentliche Entwicklungshilfe“ verbuchte) Hilfe für die Entwicklungsländer und die öffentliche Hilfe für die Transitionsländer (sog. „fortgeschrittenere Entwicklungsländer“ und Transitionsländer, d.h. ein Teil der früheren Ostblockstaaten). Die vollständige DAC-Liste der Entwicklungs- und Transitionsländer ist am Schluss des Jahrbuchs aufgeführt.

Grafik 7 : Entwicklung der öffentlichen Hilfe der DAC-Länder, 1986- 2001 in Milliarden laufender Dollar (linke Achse) und in Prozent des BVE (rechte Achse)

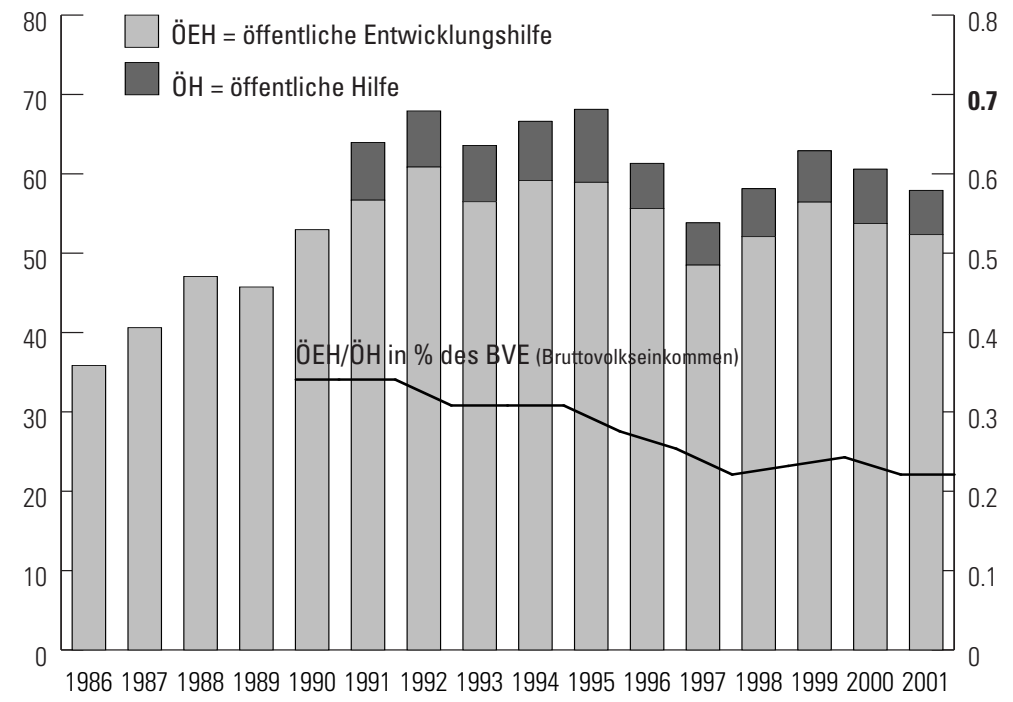

Quellen: OECD, DAC-Berichte, Coopération pour le développement.

Die öffentliche Entwicklungshilfe der DAC-Länder ist von 1999 bis 2001 um 7,3 Prozent zurückgegangen. Sie ist von 0,33 Prozent des Bruttovolkseinkommens (BVE) im Jahr 1992 auf 0,22 Prozent des BVE im Jahr 2001 abgesunken. Damit entfernt sich die öffentliche Entwicklungshilfe immer mehr vom Ziel der Vereinten Nationen (0,7\% des BVE). Die Schweiz hat sich diesem Ziel niemals verschrieben, jedoch hatte sie 1992 auf der Konferenz von Rio erklärt und in den letzten Jahren wiederholt, dass sie eine öffentliche Entwicklungshilfe in Höhe von 0,4 Prozent des BVE erreichen möchte. 
Zur Grafik 8 (Schweiz) ist darauf zu verweisen, dass die öffentliche Entwicklungshilfe der Schweiz 1992, d.h. im Jahr des Beitritts der Schweiz zu den Bretton-Woods-Institutionen, aussergewöhnlich hoch war. Ohne Berücksichtigung des Jahres 1992 stellt man fest, dass die schweizerische öffentliche Entwicklungshilfe von 1986 bis 1995 gestiegen ist und seit 1995 zurückgeht. In Prozent des BVE stagniert die öffentliche Entwicklungshilfe der Schweiz seit 1994.

Die Hilfe für die Transitionsländer (früherer Ostblock; öffentliche Hilfe - ÖH) bleibt relativ bescheiden. Hierzu ist jedoch zu vermerken, dass die Hilfe für die Staaten des früheren Jugoslawiens, welche seit Anfang der 90er Jahre sehr hoch ist, nicht im statistischen Aggregat „öffentliche Hilfe für die Transitionsländer“, sondern in der öffentliche Entwicklungshilfe enthalten ist, da diese Staaten in die Gruppe der Entwicklungsländer eingestuft wurden. Im 4. Teil der Statistiken ist die Höhe der Hilfe für die Staaten Ex-Jugoslawiens ersichtlich.

Grafik 8 : Entwicklung der öffentlichen Hilfe der Schweiz, 1986-2001

in Milliarden laufender Franken (linke Achse) und in Prozent des BVE (rechte Achse)

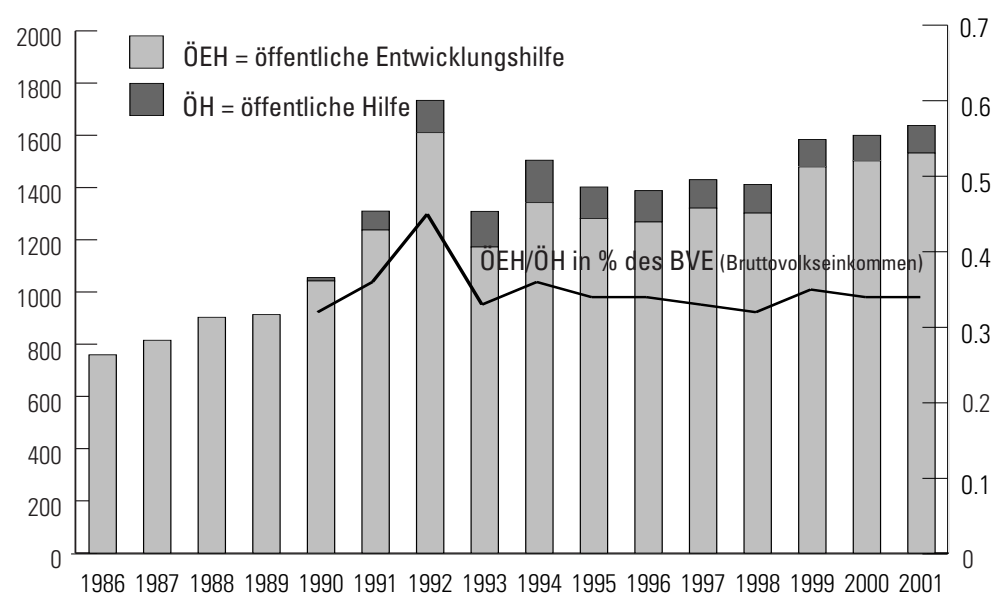

Quellen: OECD, DAC-Berichte, Coopération pour le développement.

Die Grafiken 9 und 10 auf der nächsten Seite zeigen, dass im Jahr 2001 nur fünf DAC-Länder das von den Vereinten Nationen festgesetzte Ziel von 0,7 Prozent des Bruttovolkseinkommens überschritten haben, und zwar: Dänemark, Norwegen, die Niederlande, Luxemburg und Schweden. Das gewogene Mittel der DAC-Länder lag 2001 bei 0,22 Prozent des BVE. 
Grafik 9: Öffentliche Netto-Entwicklungshilfe der DAC-Mitgliedsländer, 2001 (Milliarden Dollar)

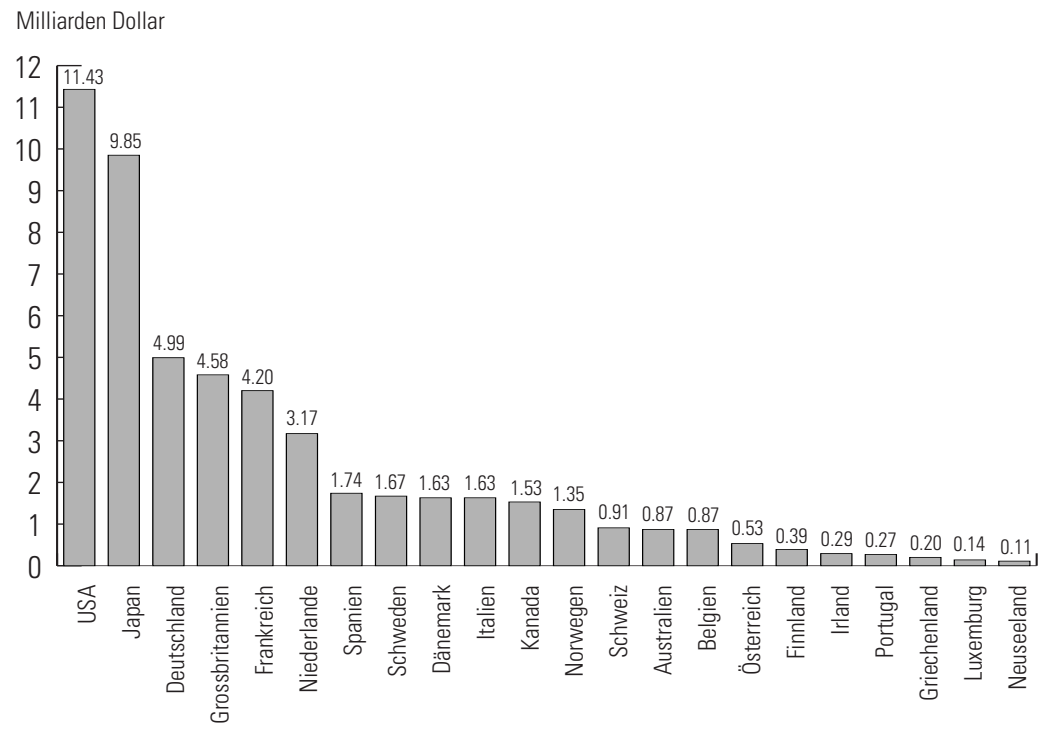

Grafik 10 : Öffentliche Netto-Entwicklungshilfe der DAC-Mitgliedsländer 2001 (in Prozent des BVE)

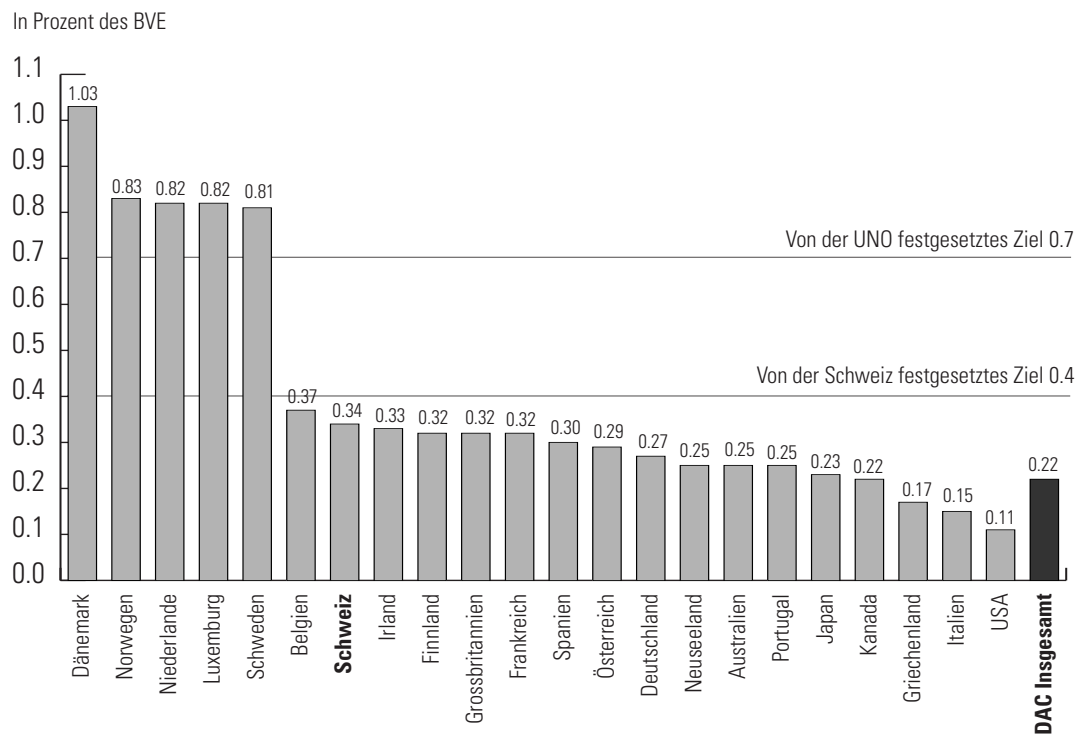

Quelle: OECD, DAC-Berichte, Coopération pour le développement. 


\section{Geografische Aufteilung der bilateralen Hilfe}

(Tabellen 3.4 und 4.2)

Die geografische Aufteilung der bilateralen Hilfeleistungen der Schweiz nach Kontinenten ist derjenigen der Leistungen sämtlicher DAC-Länder recht ähnlich. Die Unterschiede zwischen den Geberländern sind grösser, wenn man die wichtigsten Hilfeempfängerländer betrachtet. Die Grossmächte unterstützen vor allem Regionen und Länder von strategischer Bedeutung oder Märkte, die in vollem Aufschwung begriffen sind. Die Schweiz misst den am meisten benachteiligten Ländern Priorität bei.

Die Vereinten Nationen empfehlen den Geberländern, den am wenigsten fortgeschrittenen Ländern (LDC) Hilfeaufwendungen von mindestens 0,15 Prozent ihres BVE zukommen zu lassen. 2001 machte die Hilfe der Schweiz für die LDC 0,10 Prozent des BVE aus, während der Durchschnitt sämtlicher DAC-Länder bei 0,06 Prozent des BVE lag.

Wir verweisen auch auf den 4. Teil der Statistiken bezüglich der geografischen Aufteilung der Hilfe nach Regionen und Kontinenten.

\section{Vergleichende Entwicklung der verschiedenen Komponenten der Hilfe (Grafik 11)}

Geht man von einem Index 100 für 1988 aus, so ist aus der Grafik 11 zu ersehen, dass die Hilfe der Schweizer Gemeinden von 1988 bis 2001 stagniert hat, bzw. zurückgegangen ist. Die Hilfe der Schweizer Kantone war viel unbeständiger, was zum Grossteil auf die starken Schwankungen der Leistungen einiger Kantone zurückzuführen ist. Insgesamt bekam die Hilfe der Kantone und Gemeinden die direkten Folgen der Budgetkürzungen zu spüren. Die Hilfe der Nichtregierungsorganisationen (NRO), die aus Spendensammlungen dieser Organisationen finanziert wird, hat etwas stärker als die gesamte öffentliche Entwicklungshilfe von Bund, Kantonen und Gemeinden zugenommen.

Grafik 11 : Entwicklung der öffentlichen Entwicklungshilfe/Hilfe und der Hilfe der NRO, 1988-2001 (Index 1988: 100)

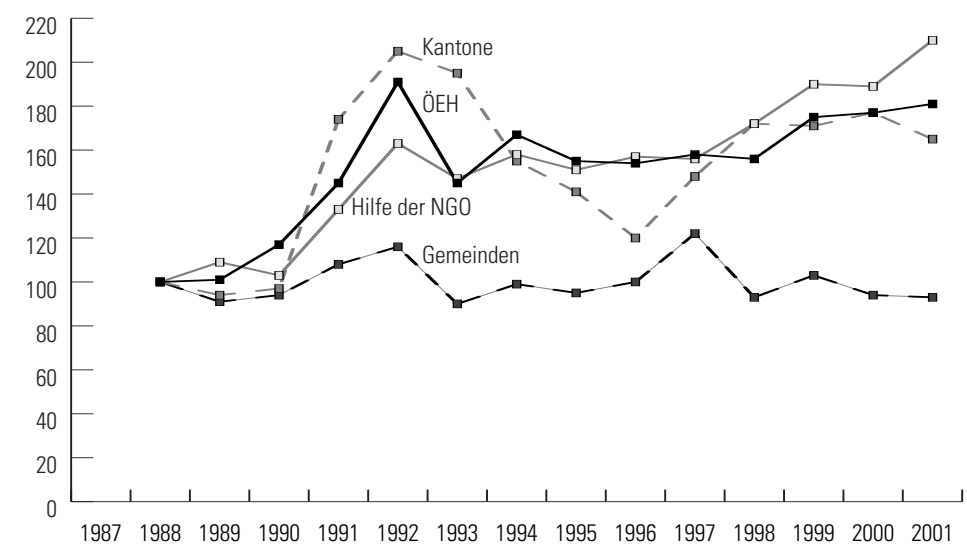

Quellen: Swissaid, Schweizerische Hilfe für Entwicklungsländer, 1988-1990, Bern; iuéd/DEZA, Schweizerische Hilfe für Entwicklungsländer und Oststaaten, Ausgaben 1991-2000, Genf; DEZA, Statistischer Dienst. 


\section{Hilfe der NRO}

Wie die nachstehende Grafik 12 zeigt, weist die aus Eigenmitteln finanzierte Hilfe der Nichtregierungsorganisationen seit 1985 einen deutlichen Aufwärtstrend auf. Die Grafik umfasst nur die aus Eigenmitteln der Organisationen finanzierten Beträge, nicht aber die Projekte der Entwicklungszusammenarbeit, die von den NRO verwaltet, aber aus Beiträgen von Kantonen, Gemeinden oder von der DEZA finanziert werden. Die unten aufgeführten Angaben entsprechen den in Entwicklungs- und Transitionsländern zur Finanzierung von Projekten der Entwicklungszusammenarbeit oder von humanitären Aktionen aufgewandten Beträgen. Sie entsprechen somit auch nicht dem Betrag der Sammlungen der Hilfswerke in der schweizerischen Öffentlichkeit, da ein Teil der Sammlungen zur Finanzierung der Verwaltungskosten der NRO, bzw. ihrer Marketingspesen und der mit den Sammelkampagnen verbundenen Aufwendungen dient.

Grafik 12: Entwicklung der Hilfe der NRO in der Schweiz, 1985-2001

(in Millionen laufender Franken)

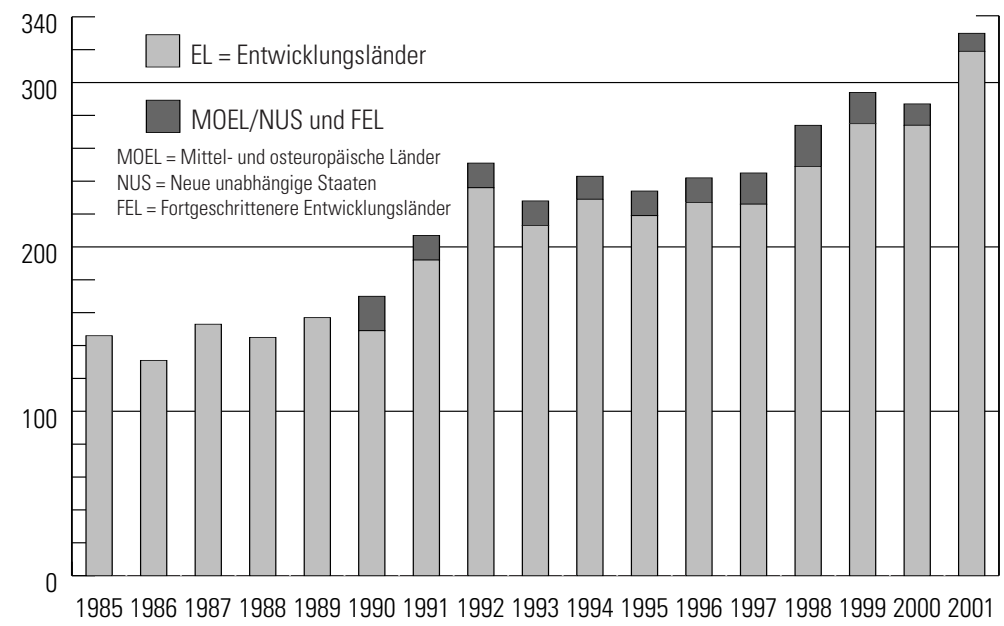

Quellen: Swissaid, Schweizerische Hilfe für Entwicklungsländer, 1988-1990, Bern; iuéd/DEZA, Schweizerische Hilfe für Entwicklungsländer und Oststaaten, Ausgaben 1991-2000, Genf; DEZA, Statistischer Dienst. 
3.1. Allgemeine Übersicht über die öffentliche und private Hilfe der Schweiz

Nettoauszahlungen der Schweiz

A. Öffentliche Entwicklungshilfe für die Entwicklungsländer

(1. Teil der DAC-Liste) ${ }^{1}$

\begin{tabular}{lrrrrrrr}
\hline \multirow{2}{*}{ Art der Beiträge } & $\mathbf{1 9 9 9}$ & $\mathbf{2 0 0 0}$ & $\mathbf{2 0 0 1}$ & $\mathbf{1 9 9 8}$ & $\mathbf{2 0 0 0}$ & $\mathbf{2 0 0 1}$ \\
\cline { 2 - 8 } & \multicolumn{3}{c}{ In Millionen Franken } & \multicolumn{3}{c}{ In \% des Gesamtbetrags } \\
\hline Bilaterale Hilfe & $\mathbf{1 1 0 1}$ & $\mathbf{1 0 6 0}$ & $\mathbf{1 0 8 6}$ & $\mathbf{7 4}$ & $\mathbf{7 0}$ & $\mathbf{7 1}$ \\
\hline Geschenke & 1094 & 1026 & 1085 & 74 & 68 & $\mathbf{7 1}$ \\
\hline Darlehen & 8 & 34 & 2 & 1 & 2 & 0 \\
\hline Multilaterale Hilfe & $\mathbf{3 7 9}$ & $\mathbf{4 4 4}$ & $\mathbf{4 4 4}$ & $\mathbf{2 6}$ & $\mathbf{3 0}$ & $\mathbf{2 9}$ \\
\hline Insgesamt & $\mathbf{1 4 8 0}$ & $\mathbf{1 5 0 4}$ & $\mathbf{1 5 3 0}$ & $\mathbf{1 0 0}$ & $\mathbf{1 0 0}$ & $\mathbf{1 0 0}$ \\
\hline
\end{tabular}

B. Öffentliche Hilfe für die mittel- und osteuropäischen Länder und die fortgeschritteneren Entwicklungsländer (2. Teil der DAC-Liste) ${ }^{1}$

\begin{tabular}{lcccrrrr}
\hline \multirow{2}{*}{ Art der Beiträge } & $\mathbf{1 9 9 9}$ & $\mathbf{2 0 0 0}$ & $\mathbf{2 0 0 1}$ & $\mathbf{1 9 9 8}$ & $\mathbf{2 0 0 0}$ & $\mathbf{2 0 0 1}$ \\
\cline { 2 - 8 } & \multicolumn{2}{c}{ In Millionen Franken } & & \multicolumn{2}{c}{ In \% des Gesamtbetrags } \\
\hline Bilaterale Hilfe & $\mathbf{9 5}$ & $\mathbf{9 8}$ & $\mathbf{9 3}$ & $\mathbf{9 1}$ & $\mathbf{1 0 0}$ & $\mathbf{8 9}$ \\
\hline Geschenke & 93 & 96 & 89 & 90 & 98 & 85 \\
\hline Darlehen & 2 & 2 & 3 & 1 & 2 & 3 \\
\hline Multilaterale Hilfe & $\mathbf{9}$ & $\mathbf{0}$ & $\mathbf{1 2}$ & $\mathbf{9}$ & $\mathbf{0}$ & $\mathbf{1 1}$ \\
\hline Insgesamt & $\mathbf{1 0 4}$ & $\mathbf{9 8}$ & $\mathbf{1 0 5}$ & $\mathbf{1 0 0}$ & $\mathbf{1 0 0}$ & $\mathbf{1 0 0}$ \\
\hline
\end{tabular}

C. Geschenke privater Hilfsorganisationen, in Millionen Franken

\begin{tabular}{lrrr}
\hline Art der Beiträge & $\mathbf{1 9 9 9}$ & $\mathbf{2 0 0 0}$ & $\mathbf{2 0 0 1}$ \\
\hline Geschenke für die Entwicklungsländer & 275 & 274 & 304 \\
\hline Geschenke für die Länder des 2. Teils der DAC-Liste & 19 & 14 & 12 \\
\hline
\end{tabular}

Quelle: <www.oecd.org/dac>, Statistischer Teil, Tabelle 13e, 38e und 39e.

1 Siehe Länderklassifikation (1. und 2. Teil der DAC-Liste) am Schluss des Jahrbuchs. 


\section{DAC-Definitionen für die Tabellen 3.1. und 3.2.}

- Öffentliche Entwicklungshilfe: Darlehen oder Geschenke an die im 1. Teil der DAC-Liste aufgeführten Länder (Entwicklungsländer). Siehe Liste am Schluss des Jahrbuchs.

Die öffentliche Entwicklungshilfe wird vom öffentlichen Sektor mit dem Ziel aufgebracht, zur Erleichterung der wirtschaftlichen Entwicklung und zur Verbesserung der Lebensbedingungen zu liberalen Finanzbedingungen beizutragen (bei Darlehen muss der liberale Anteil mindestens 25\% betragen).

」 Öffentliche Hilfe: Beiträge, die den Kriterien der öffentlichen Entwicklungshilfe entsprechen, deren Empfänger aber im 2. Teil der DAC-Liste aufgeführt sind (Transitionsländer, d.h. Länder Mittel- und Osteuropas und fortgeschrittenere Entwicklungsländer).

- Bilaterale Hilfe: Direktzahlungen eines Geberlandes an ein Empfängerland der Hilfe. Zahlungen an multilaterale Organisationen für ein vom Geberland ausgewähltes spezifisches Projekt sind in der bilateralen Hilfe enthalten. In der Schweiz werden die Beiträge an das IKRK ebenfalls unter der bilateralen Hilfe verbucht.

- Multilaterale Hilfe: Zahlungen eines Geberlandes, die über eine im Entwicklungsbereich tätige internationale Organisation geleitet werden. Der Beitrag wird als multilateral bezeichnet, wenn er mit den Beiträgen anderer Mitgliedsländer der Organisation zusammenfällt und diese über die Zuwendung der Mittel allein entscheidet.

Quelle: OCDE, Coopération pour le développement, Rapport 2000. 
3.2. Allgemeine Übersicht über die öffentliche und private Hilfe der DAC-Länder

\section{Nettoauszahlungen der DAC-Mitgliedsländer}

A. Öffentliche Entwicklungshilfe für die Entwicklungsländer (1. Teil der DAC-Liste) ${ }^{1}$

\begin{tabular}{lrrrrrrr}
\hline \multirow{2}{*}{ Art der Beiträge } & $\mathbf{1 9 9 9}$ & $\mathbf{2 0 0 0}$ & $\mathbf{2 0 0 1}$ & $\mathbf{1 9 9 8}$ & $\mathbf{2 0 0 0}$ & $\mathbf{2 0 0 1}$ \\
\cline { 2 - 8 } & \multicolumn{3}{c}{ In Millionen Dollars } & \multicolumn{2}{c}{ In \% des Gesamtbetrags } \\
\hline Bilaterale Hilfe & $\mathbf{3 7 8 7 8}$ & $\mathbf{3 6 0 4 9}$ & $\mathbf{3 5 0 2 2}$ & $\mathbf{6 7}$ & $\mathbf{6 7}$ & $\mathbf{6 7}$ \\
\hline Geschenke & 33922 & 33028 & 33409 & 60 & 61 & 64 \\
\hline Darlehen & 3956 & 3021 & 1613 & 7 & 6 & 3 \\
\hline Multilaterale Hilfe & $\mathbf{1 8 5 5 1}$ & $\mathbf{1 7 6 8 5}$ & $\mathbf{1 7 3 1 4}$ & $\mathbf{3 3}$ & $\mathbf{3 3}$ & $\mathbf{3 3}$ \\
\hline Insgesamt & $\mathbf{5 6 4 2 9}$ & $\mathbf{5 3 7 3 4}$ & $\mathbf{5 2 3 3 6}$ & $\mathbf{1 0 0}$ & $\mathbf{1 0 0}$ & $\mathbf{1 0 0}$ \\
\hline
\end{tabular}

B. Öffentliche Hilfe für die mittel- und osteuropäischen Länder und die fortgeschritteneren Entwicklungsländer (2. Teil der DAC-Liste) ${ }^{1}$

\begin{tabular}{lrrrrrr}
\hline \multirow{2}{*}{ Art der Beiträge } & $\mathbf{1 9 9 9}$ & $\mathbf{2 0 0 0}$ & $\mathbf{2 0 0 1}$ & $\mathbf{1 9 9 8}$ & $\mathbf{2 0 0 0}$ & $\mathbf{2 0 0 1}$ \\
\cline { 2 - 8 } & \multicolumn{3}{c}{ In Millionen Dollars } & \multicolumn{2}{c}{ In \% des Gesamtbetrags } \\
\hline Bilaterale Hilfe & $\mathbf{4 8 5 2}$ & $\mathbf{4 8 8 0}$ & $\mathbf{3 6 3 2}$ & $\mathbf{7 5}$ & $\mathbf{7 1}$ & $\mathbf{6 5}$ \\
\hline Geschenke & 4763 & 4944 & 3967 & 74 & 72 & $\mathbf{7 1}$ \\
\hline Darlehen & 89 & -64 & -335 & 1 & -1 & -6 \\
\hline Multilaterale Hilfe & $\mathbf{1 6 1 6}$ & $\mathbf{1 9 7 2}$ & $\mathbf{1 9 4 2}$ & $\mathbf{2 5}$ & $\mathbf{2 9}$ & $\mathbf{3 5}$ \\
\hline Insgesamt & $\mathbf{6 4 6 8}$ & $\mathbf{6 8 5 2}$ & $\mathbf{5 5 7 4}$ & $\mathbf{1 0 0}$ & $\mathbf{1 0 0}$ & $\mathbf{1 0 0}$ \\
\hline
\end{tabular}

C. Geschenke privater Hilfsorganisationen, in Millionen Dollar

\begin{tabular}{llll}
\hline Art der Beiträge & $\mathbf{1 9 9 9}$ & $\mathbf{2 0 0 0}$ & $\mathbf{2 0 0 1}$ \\
\hline Geschenke für die Entwicklungsländer & 6715 & 6935 & 7289 \\
\hline Geschenke für die Länder des 2. Teils der DAC-Liste & 2232 & 2524 & 3151 \\
\hline
\end{tabular}

Quelle: <www.oecd.org/dac>, Statistischer Teil, Tabelle 13e, 38e und 39e.

1 Siehe Länderklassifikation (1. und 2. Teil der DAC-Liste) am Schluss des Jahrbuchs. 


\section{3. Öffentliche und private Hilfe in der Schweiz für die Entwicklungs- und Transitionsländer, in Tausend Franken}

\begin{tabular}{|c|c|c|c|c|c|c|}
\hline & \multicolumn{3}{|c|}{2000} & \multicolumn{3}{|c|}{2001} \\
\hline & Bilat. & Multilat. & Insgesamt & Bilat. & Multilat. & Insgesamt \\
\hline Hilfe für Entwicklungsländer 1 & 1329397 & 445286 & 1774683 & 1407585 & 443817 & 1851402 \\
\hline Öffentliche Entwicklungshilfe 1 & 1057370 & 445286 & 1532067 & 1088250 & 443817 & 1532067 \\
\hline DEZA & 746483 & 369196 & 1174238 & 809019 & 365219 & 1174238 \\
\hline $\begin{array}{l}\text { Entwicklungs- } \\
\text { zusammenarbeit }\end{array}$ & 418559 & 343544 & 762103 & 447847 & 336680 & 784527 \\
\hline Humanitäre Hilfe & 226828 & 25652 & 252480 & 217709 & 28539 & 246248 \\
\hline Ostzusammenarbeit & 46721 & - & 46721 & 64865 & - & 64865 \\
\hline $\begin{array}{l}\text { Programmbeiträge an } \\
\text { Schweizer NRO }\end{array}$ & 54265 & - & 54265 & 54641 & - & 54641 \\
\hline seco & 166576 & 27469 & 194045 & 149181 & 25859 & 175040 \\
\hline $\begin{array}{l}\text { Entwicklungs- } \\
\text { zusammenarbeit }\end{array}$ & 102288 & 27469 & 129757 & 93653 & 25859 & 119511 \\
\hline Ostzusammenarbeit & 64288 & - & 64288 & 55529 & - & 55529 \\
\hline$B F F$ & 79464 & - & 79464 & 73709 & - & 73709 \\
\hline$E D A$ & 19400 & 10416 & 29815 & 20211 & 10673 & 30884 \\
\hline$E F V$ & 5900 & 15204 & 21104 & - & 15694 & 15694 \\
\hline BUWAL & 1444 & 12863 & 14307 & 3225 & 15347 & 18572 \\
\hline$V B S$ & - & - & - & 250 & - & 250 \\
\hline$\overline{B A G}$ & 40 & 6836 & 6876 & 100 & 7225 & 7325 \\
\hline$B L W$ & 2527 & 3138 & 5665 & - & 3654 & 3654 \\
\hline$B B W$ & 4225 & - & 4225 & 3342 & - & 3342 \\
\hline$S M A$ & - & 25 & 25 & - & 26 & 26 \\
\hline UVEK & - & 54 & 54 & - & 28 & 28 \\
\hline BVET & - & 85 & 85 & - & 92 & 92 \\
\hline Verwaltungskosten, nicht aufgeteil & ilt 9024 & - & 9024 & 6750 & - & 6750 \\
\hline Kantone & 14159 & - & 14159 & 13437 & - & 13437 \\
\hline Gemeinden & 8128 & - & 8128 & 9027 & - & 9027 \\
\hline $\begin{array}{l}\text { Hilfe der NRO, } \\
\text { aus Eigenmitteln }\end{array}$ & 272027 & - & 272027 & 319335 & - & 319335 \\
\hline
\end{tabular}

BFF : Bundesamt für Flüchtlinge

EDA : Eidg. Departement für auswärtige Angelegenheiten

EFV : Eidg. Finanzverwaltung

BUWAL: Bundesamt für Umwelt, Wald und Landschaft

VBS : Eidg. Departement für Verteidigung, Bevölkerungsschutz und Sport

BAG : Bundesamt für Gesundheitswesen

BLW : Bundesamt für Landwirtschaft

BBW : Bundesamt für Bildung und Wissenschaft

SMA: Schweizerische Metereologische Anstalt

UVEK : Eidg. Departement für Umwelt, Verkehr, Energie und Kommunikation

BVET: Bundesamt für Veterinärwesen 


\section{3. Öffentliche und private Hilfe in der Schweiz für die Entwicklungs- und Transitionsländer, in Tausend Franken}

\begin{tabular}{|c|c|c|c|c|c|c|}
\hline & & 2000 & & & 2001 & \\
\hline & Bilat. & Multilat. & Insgesamt & Bilat. & Multilat. & Insgesamt \\
\hline Hilfe für Transitionssländer $^{1}$ & 111688 & 10608 & 122296 & 104847 & 12554 & 117401 \\
\hline Öffentliche Entwicklungshilfe & 97758 & 10608 & 108367 & 93557 & 12554 & 106111 \\
\hline DEZA & 40957 & - & 40957 & 45846 & - & 45846 \\
\hline $\begin{array}{l}\text { Entwicklungs- } \\
\text { zusammenarbeit }\end{array}$ & 1386 & - & 1386 & 1634 & - & 1634 \\
\hline Humanitäre Hilfe & 9607 & - & 9607 & 9410 & - & 9410 \\
\hline Ostzusammenarbeit & 29904 & - & 29904 & 34726 & - & 34726 \\
\hline $\begin{array}{l}\text { Programmbeiträge an } \\
\text { Schweizer NRO }\end{array}$ & 60 & - & 60 & 75 & - & 75 \\
\hline seco & 53038 & 7798 & 60836 & 43992 & 9029 & 53021 \\
\hline Entwicklungszusammenarbe & eit $\quad-$ & - & - & 16 & - & 16 \\
\hline Ostzusammenarbeit & 53038 & 7798 & 60836 & 43976 & 9029 & 53005 \\
\hline$E D A$ & 364 & - & 364 & 518 & - & 518 \\
\hline BUWAL & - & 2810 & 2810 & - & 3525 & 3525 \\
\hline$B A G$ & - & - & - & - & - & - \\
\hline$\overline{B B W}$ & 1469 & - & 1469 & 1361 & - & 1361 \\
\hline SMA & - & - & - & - & - & - \\
\hline Kantone & 697 & - & 697 & 911 & - & 911 \\
\hline Gemeinden & 1233 & - & 1233 & 930 & - & 930 \\
\hline $\begin{array}{l}\text { Hilfe der NRO } \\
\text { aus Eigenmitteln }\end{array}$ & 12937 & - & 12937 & 11290 & - & 11290 \\
\hline $\begin{array}{l}\text { Andere Beiträge } \\
\text { des öffentlichen Sektors }\end{array}$ & 12694 & - & 12694 & 11825 & - & 11825 \\
\hline seco & 12694 & - & 12694 & 11825 & - & 11825 \\
\hline $\begin{array}{l}\text { Entwicklungs- } \\
\text { zusammenarbeit }\end{array}$ & 12694 & - & 12694 & 10825 & - & 10825 \\
\hline Ostzusammenarbeit & - & - & - & 1000 & - & 1000 \\
\hline
\end{tabular}

Quelle: DEZA, Statistischer Dienst.

Hilfe für die Oststaaten und die fortgeschritteneren Entwicklungsländer.

2 Andere Beiträge des öffentlichen Sektors, die nicht den Bedingungen der öffentlichen Entwicklungshilfe/öffentlichen Hilfe entsprechen. 


\section{4. Öffentliche Entwicklungshilfe}

Die zwanzig Hauptempfängerländer (2001)

Vergleich zwischen den Nettoauszahlungen der Schweiz und den Nettoauszahlungen sämtlicher DAC-Mitgliedsländer

Auszahlungen der Schweiz 2001 ${ }^{\text {p }}$

Auszahlungen der Mitgliedsländer des OECD-Entwicklungshilfeausschusses $2001^{\mathrm{p}}$

\begin{tabular}{|c|c|c|c|c|c|c|c|}
\hline Land & Mio Fr. & $\%$ & $\sum \%$ & Land & Mio Fr. & $\%$ & $\sum \%$ \\
\hline 1. Jugoslawien, Bundesrep. & p. 57,7 & 5,3 & 5,3 & 1. Pakistan & 3269 & 4,0 & 4,0 \\
\hline 2. Mosambik & 39,4 & 3,6 & 8,8 & 2. Indien & 2877 & 3,5 & 7,4 \\
\hline 3. Indien & 37,2 & 3,4 & 12,2 & 3. Indonesien & 2532 & 3,1 & 10,5 \\
\hline 4. Div. Staaten Ex-Jugosl. & 35,1 & 3,2 & 15,4 & 4. China & 2463 & 3,0 & 13,5 \\
\hline 5. Tansania & 28,7 & 2,6 & 18,0 & 5. Vietnam & 2420 & 2,9 & 16,4 \\
\hline 6. Burkina Faso & 21,5 & 2,0 & 20,0 & 6. Jugoslawien, Bundesrep & p. 2203 & 2,7 & 19,1 \\
\hline 7. Bosnien-Herzegowina & 21,1 & 1,9 & 21,9 & 7. Ägypten & 2117 & 2,6 & 21,6 \\
\hline 8. Nepal & 20,9 & 1,9 & 23,8 & 8. Tansania & 2081 & 2,5 & 24,1 \\
\hline 9. Bangladesch & 20,7 & 1,9 & 25,7 & 9. Äthiopien & 1821 & 2,2 & 26,3 \\
\hline 10. Vietnam & 18,3 & 1,7 & 27,4 & 10. Bangladesch & 1727 & 2,1 & 28,4 \\
\hline 11. Bolivien & 18,0 & 1,6 & 29,0 & 11. Mosambik & 1577 & 1,9 & 30,3 \\
\hline 12. Peru & 15,2 & 1,4 & 30,4 & 12. Nicaragua & 1566 & 1,9 & 32,2 \\
\hline 13. Pakistan & 15,0 & 1,4 & 31,7 & 13. Gebiete unter paläst. Verv & rw. 1459 & 1,8 & 34,0 \\
\hline 14. Südafrika & 13,6 & 1,2 & 33,0 & 14. Uganda & 1320 & 1,6 & 35,6 \\
\hline 15. Afghanistan & 12,6 & 1,1 & 34,1 & 15. Bolivien & 1229 & 1,5 & 37,1 \\
\hline 16. Niger & 12,4 & 1,1 & 35,3 & 16. Honduras & 1143 & 1,4 & 38,5 \\
\hline 17. Tschad & 12,0 & 1,1 & 36,4 & 17. Ghana & 1100 & 1,3 & 39,8 \\
\hline 18. Tadschikistan & 11,8 & 1,1 & 37,4 & 18. Bosnien-Herzegowina & 1078 & 1,3 & 41,1 \\
\hline 19. Ekuador & 11,7 & 1,1 & 38,5 & 19. Philippinen & 973 & 1,2 & 42,3 \\
\hline 20. Gebiete unter paläst. Verw. & w. 10,7 & 1,0 & 39,5 & 20. Marokko & 871 & 1,1 & 43,3 \\
\hline Andere Länder & 256,6 & 23,3 & 62,8 & Andere Länder & 37045 & 42,5 & 83,6 \\
\hline Nicht angegeben ${ }^{1}$ & 408,8 & 37,2 & 100,0 & Nicht angegeben $^{1}$ & 14307 & 16,4 & 100,0 \\
\hline Insgesamt $^{2}$ & 1099,1 & 100,0 & & Insgesamt $^{3}$ & 87180 & 100,0 & \\
\hline
\end{tabular}

Quellen:

- für die Schweiz: DEZA, Statistischer Dienst.

- für das DAC: www.oecd.org/dac, Statistischer Teil, Tabelle 25e.

Anmerkung: Die Aufteilung nach Ländern zeigt, dass die Grossmächte vor allem die strategisch wichtigen Gebiete, ferner Länder, deren Märkte in vollem Aufschwung sind, oder die früheren Kolonien unterstützen. Die Schweiz hingegen räumt denjenigen Ländern, die eine humanitäre Krise durchmachen oder zu den ärmsten Ländern gehören, Priorität ein.

p provisorische Zahlen.

1 Die Bestimmung dieser Auszahlungen ist nicht aufgeführt, da sie mehrere Länder zugleich betreffen.

2 Bilaterale Hilfe insgesamt.

3 Gesamte bilaterale Hilfe der DAC-Länder, der multilateralen Organisationen und der arabischen Länder. Somit unterscheidet sich dieser Gesamtbetrag vom Betrag der Tabelle 3.1.A. 


\subsection{Multilaterale Beiträge öffentlicher Entwicklungshilfe und öffentlicher Hilfe nach Organisationen, in Tausend Franken}

\begin{tabular}{|c|c|c|}
\hline Organisationen & 2000 & 2001 \\
\hline Multilaterale öffentliche Entwicklungshilfe & 445286 & 443817 \\
\hline Sonderorganisationen der Vereinten Nationen & 134225 & 139917 \\
\hline UNDP, UN-Entwicklungsprogramm & 52000 & 52000 \\
\hline UNICEF, Kinderhilfswerk der Vereinten Nationen & 17000 & 17000 \\
\hline UNHCR, UN-Hochkommissariat für Flüchtlinge & 13000 & 13000 \\
\hline UNFPA, Bevölkerungsfonds der Vereinten Nationen & 11500 & 12000 \\
\hline UNWRA, UN-Hilfswerk für die Palästinaflüchtlinge im Nahen Osten & 9421 & 11100 \\
\hline UNEP, UN-Umweltprogramm & 4468 & 4897 \\
\hline UNAIDS, HIV/AIDS-Programm der Vereinten Nationen & 2200 & 4000 \\
\hline WHO, Spezialprogramme & 4954 & 3619 \\
\hline OCHA, UN-Büro für die Koordination humanitärer Angelegenheiten & 298 & 1902 \\
\hline UNIDO, UN-Organisation für industrielle Enwicklung & 1793 & 1793 \\
\hline WFP, Weltrernährungsprogramm & 1500 & 1499 \\
\hline IOM, Internationale Organisation für Migration & 1041 & 1038 \\
\hline UNDCP, Internationales Drogenbekämpfungsprogramm & 847 & 847 \\
\hline UNIFEM, UN-Entwicklungsfonds für die Frauen & 800 & 800 \\
\hline UNV, UN-Freiwilligenprogramm & 500 & 600 \\
\hline UNCCD, UN-Konvention zur Bekämpfung der Wüstenbildung & 325 & 518 \\
\hline UNRISD, Forschungsinstitut der Vereinten Nationen für soziale Entwicklung & 96 & 96 \\
\hline UNITAR, Ausbildungs- und Forschungsinstitut der Vereinten Nationen & 95 & 95 \\
\hline UNFCCC, UN-Rahmenkonvention über Klimaänderungen & 120 & - \\
\hline Allgemeine Beiträge an UN-Organisationen (teilweise verbuchbare Beträge) & 12267 & 13112 \\
\hline Entwicklungsfinanzierungsinstitutionen und ihre Sonderfonds & 290466 & 267448 \\
\hline IDA, Internationale Entwicklungsorganisation & 140000 & 140000 \\
\hline AfDF, Afrikanischer Entwicklungsfonds & 92067 & 46034 \\
\hline HIPC, Fonds der IDA für hochverschuldete arme Länder & 25000 & 23462 \\
\hline IFAD, Internationaler Fonds für landwirtschaftliche Entwicklung & 151 & 17718 \\
\hline AsDF, Asiatischer Entwicklungsfonds & 10631 & 13926 \\
\hline PRGF, Poverty Reduction and Growth Facility des IWF & 8204 & 8515 \\
\hline IWF, Treuhandsfonds des Internationalen Währungsfonds & 7000 & 7179 \\
\hline Weltbank & 3285 & 3423 \\
\hline MIGA, Multilaterale Investitionsgarantieagentur & - & 3102 \\
\hline AfDB, Afrikanische Entwicklungsbank (ordentliches Kapital) & 1964 & 1918 \\
\hline IDB, Interamerikanische Entwicklungsbank & 1427 & 1570 \\
\hline AsDB, Asiatische Entwicklungsbank & 737 & 601 \\
\hline
\end{tabular}




\begin{tabular}{lrr}
\hline Organisationen & $\mathbf{2 0 0 0}$ & $\mathbf{2 0 0 1}$ \\
\hline $\begin{array}{l}\text { Andere multilaterale Organisationen } \\
\text { CGIAR, Beratungsgruppe für internationale Agrarforschung }\end{array}$ & 20594 & 36452 \\
\hline $\begin{array}{l}\text { GEF, Globaler Umweltfonds (zu 75\% unter öffentlicher } \\
\text { Entwiclungshilfe verbuchbare Beiträge }\end{array}$ & 3019 & 11021 \\
\hline GF-ATM, Globaler Fonds zur Bekämpfung von AIDS, Tuberkulose und Malaria & 8432 & 10575 \\
\hline AIF, Agence intergouvernementale de la Francophonie & - & 4950 \\
\hline Protokoll von Montreal über den Schutz der Ozonschicht & 3124 & 4325 \\
\hline $\begin{array}{l}\text { IUCN, Internationale Union für die Erhaltung der Natur } \\
\text { und der natürlichen Hilfsquellen }\end{array}$ & 1190 & 3148 \\
\hline ICDDR, International Centre for Research on Diarrhea Diseases & - & 1198 \\
\hline CABI, Int. Zentrum für Landwirtschaft und Biowissenschaften & 51 & 850 \\
\hline CITES, Übereinkommen über den internationalen Handel mit gefährdeten Arten & 75 & 118 \\
\hline ICAC, Internationales Baumwollberatungskomitee & 21 & 81 \\
\hline ACCT, Agentur für kulturelle und technische Zusammenarbeit & 4233 & - \\
\hline WCD, Weltstaudammkommission & 450 & - \\
\hline Andere Institutionen & $\mathbf{1 0 6 0 8}$ & 162 \\
\hline $\begin{array}{l}\text { Multilaterale öffentliche Hilfe } \\
\text { (Hilfe für Oststaaten u. fortgeschritt. Entwicklungsländer) }\end{array}$ & 7798 & 9029 \\
\hline EBRD, Europäische Bank für Wiederaufbau und Entwicklung & 2811 & 3525 \\
\hline $\begin{array}{l}\text { GEF, Globaler Umweltfonds (zu 25\% unter öffentlicher } \\
\text { Hilfe verbuchbare Beiträge }\end{array}$ & & - \\
\hline
\end{tabular}

Quelle: DEZA, Statistischer Dienst. 


\subsection{Beiträge der Kantone für die Entwicklungsländer und Transitionsländer, in Tausend Franken}

A. Hilfe für die Entwicklungsländer (in der öffentlichen Entwicklungshilfe enthalten)

\begin{tabular}{|c|c|c|c|c|}
\hline Kanton & 2000 & 2001 & Fr./Einw. & $\begin{array}{r}\text { Finanz- } \\
\text { kraft }\end{array}$ \\
\hline ÖEH ${ }^{1}$ & 14159,0 & 13437,0 & 2,0 & 100 \\
\hline Genf & 3428,0 & 3405,0 & 8,5 & 130 \\
\hline Appenzell Ausserhoden & 100,0 & 124,0 & 8,3 & 59 \\
\hline Basel-Stadt & 1337,0 & 1331,0 & 7,0 & 158 \\
\hline Jura & 230,0 & 320,0 & 4,6 & 31 \\
\hline Basel-Land & 1086,0 & 1062,0 & 4,1 & 120 \\
\hline Zug & 1920,0 & 242,0 & 2,5 & 218 \\
\hline Zürich & 2802,0 & 2900,0 & 2,4 & 157 \\
\hline Neuenburg & 315,0 & 335,0 & 2,0 & 58 \\
\hline Glarus & 48,0 & 75,0 & 1,9 & 78 \\
\hline Nidwalden & 25,5 & 48,0 & 1,3 & 124 \\
\hline Waadt & 520,0 & 764,0 & 1,3 & 95 \\
\hline Aargau & 600,0 & 670,0 & 1,2 & 100 \\
\hline St. Gallen & 560,0 & 540,0 & 1,2 & 82 \\
\hline Schaffhausen & 43,0 & 71,0 & 1,0 & 106 \\
\hline Schwyz & 241,5 & 118,0 & 0,9 & 96 \\
\hline Wallis & 73,5 & 213,0 & 0,8 & 30 \\
\hline Bern & 150,0 & 695,0 & 0,7 & 66 \\
\hline Uri & 20,0 & 26,0 & 0,7 & 67 \\
\hline Tessin & 250,0 & 218,0 & 0,7 & 78 \\
\hline Obwalden & 18,8 & 21,0 & 0,7 & 40 \\
\hline Thurgau & 100,0 & 95,0 & 0,4 & 87 \\
\hline Graubünden & 42,0 & 50,0 & 0,3 & 75 \\
\hline Luzern & 150,0 & 65,0 & 0,2 & 72 \\
\hline$\underline{\text { Solothurn }}$ & 85,0 & 40,0 & 0,2 & 87 \\
\hline Freiburg & 12,0 & 8,0 & 0,0 & 52 \\
\hline Appenzell Innerhoden & 2,0 & 0,0 & 0,0 & 60 \\
\hline
\end{tabular}

${ }^{1}$ Gesamtbetrag oder Durchschnitt. 


\subsection{Beiträge der Kantone für die Entwicklungsländer und Transitionsländer, in Tausend Franken (Schluss)}

B. Hilfe für die Transitionsländer

(Hilfe für den Osten und die fortgeschritteneren Entwicklungsländer)

\begin{tabular}{lrrrr}
\hline Kanton & $\mathbf{2 0 0 0}$ & $\mathbf{2 0 0 1}$ & Fr./Einw. & $\begin{array}{r}\text { Finanz- } \\
\text { kraft }\end{array}$ \\
\hline Hilfe für die Transitionsländer & $\mathbf{6 9 6 , 7}$ & $\mathbf{9 1 1 , 0}$ & $\mathbf{0 , 1 3}$ & $\mathbf{1 0 0}$ \\
\hline Zug & 6,5 & 290,0 & 3,0 & 218 \\
\hline Appenzell Ausserhoden & - & 20,0 & 1,3 & 59 \\
\hline Basel-Stadt & 241,0 & 219,0 & 1,1 & 158 \\
\hline Jura & 25,0 & 50,0 & 0,7 & 31 \\
\hline Basel-Land & 24,2 & 77,0 & 0,3 & 120 \\
\hline Solothurn & 10,0 & 48,0 & 0,2 & 87 \\
\hline Tessin & - & 32,0 & 0,1 & 78 \\
\hline Zürich & 220,0 & 100,0 & 0,1 & 157 \\
\hline St. Gallen & 20,0 & 20,0 & 0,0 & 82 \\
\hline Bern & - & 35,0 & 0,0 & 66 \\
\hline Graubünden & 46,5 & 6,0 & 0,0 & 75 \\
\hline Schaffhausen & - & 2,0 & 0,0 & 106 \\
\hline Nidwalden & - & 1,0 & 0,0 & 124 \\
\hline Aargau & - & 10,0 & 0,0 & 100 \\
\hline Appenzell Innerhoden & - & 1,0 & 0,0 & 60 \\
\hline Freiburg & - & - & - & 52 \\
\hline Genf & - & - & - & 130 \\
\hline Glarus & - & - & - & 78 \\
\hline Luzern & - & - & - & 72 \\
\hline Neuenburg & - & - & - & 58 \\
\hline Obwalden & - & - & - & 40 \\
\hline Schwyz & - & - & - & 96 \\
\hline Thurgau & - & - & - & 87 \\
\hline Uri & -00 & - & 30 \\
\hline Wallis & - & - & - & 95 \\
\hline Waadt & - & - & - & \\
\hline & - & - & - & - \\
\hline
\end{tabular}

Quelle: DEZA, Statistischer Dienst.

Finanzkraft: Ordonnance fixant la capacité financière des cantons pour les années 2000 et 2001, du 17 novembre 1999, RO 28.12.1999, no 51, pp.3545-3548.

Bevölkerung: Bundesamt für Statistik, 1998, Neuenburg. 


\subsection{Beiträge der Gemeinden für die Entwicklungsländer und Transitionsländer}

\section{Aufwendungen 2000 und 2001, in Tausend Franken}

\begin{tabular}{|c|c|c|c|c|}
\hline \multirow[t]{2}{*}{ Kanton/Gemeinde } & \multicolumn{2}{|c|}{ Hilfe für die Entwicklungsländer } & \multicolumn{2}{|c|}{$\begin{array}{l}\text { Hilfe für die Transitionsländer } \\
\text { (Hilfe für den Osten und die fort- } \\
\text { geschritt. Entwicklungsländer) }\end{array}$} \\
\hline & 2000 & 2001 & 2000 & 2001 \\
\hline $\begin{array}{l}\text { Hilfe für die EL } \\
\text { (in der öff. EH enthalten) }\end{array}$ & 8128,0 & 9027,0 & 1232,9 & 930,0 \\
\hline Aargau & 165,9 & 86,0 & - & 5,0 \\
\hline Appenzell Ausserhoden & 5,0 & 6,0 & 2,0 & 0,0 \\
\hline Basel-Land & 253,7 & 292,0 & 166,4 & 18,0 \\
\hline davon: Reinach & 80,0 & 95,0 & & \\
\hline Allschwil & 52,0 & 57,0 & & \\
\hline Basel-Stadt & 230,0 & 200,0 & 170,0 & 170,0 \\
\hline davon: Riehen & 230,0 & 200,0 & 170,0 & 170,0 \\
\hline Bern & 637,2 & 602,0 & 235,0 & 241,0 \\
\hline davon: Bern & 240,0 & 220,0 & & \\
\hline Ittingen & & & 150,0 & 150,0 \\
\hline Muensingen & 143,0 & 136,0 & & \\
\hline Thun & 55,0 & 50,0 & & \\
\hline Muri bei Bern & 54,8 & 49,0 & & \\
\hline Freiburg & 31,6 & 29,0 & - & 2,0 \\
\hline Genf & 3620,0 & 3982,0 & 360,9 & 190,0 \\
\hline davon: Genf & 1300,0 & n.e. & 135,0 & n.e. \\
\hline Lancy & 376,0 & 430,0 & & \\
\hline Meyrin & 200,0 & 362,0 & & \\
\hline Carouge & 293,0 & 293,0 & & \\
\hline Cologny & 176,1 & 180,0 & & \\
\hline Plan les Ouates & 127,0 & 170,0 & & \\
\hline Grand-Saconnex & 10,0 & 150,0 & & \\
\hline Bernex & 121,0 & 143,0 & & \\
\hline Veyrier & 99,0 & 99,0 & & \\
\hline Thônex & 100,0 & 96,0 & & \\
\hline Chêne-Bougeries & 108,4 & 93,0 & & \\
\hline Versoix & 66,5 & 87,0 & & \\
\hline Vandouvres & 68,8 & 68,0 & & \\
\hline Troinex & 62,2 & 62,0 & & \\
\hline Chêne-Bourg & 57,0 & 60,0 & & \\
\hline Onex & 52,0 & 59,0 & & \\
\hline Vernier & 220,0 & 0,0 & & \\
\hline Glarus & - & - & & \\
\hline Graubünden & 31,5 & 52,0 & - & - \\
\hline Jura & 9,9 & 10,0 & 2,0 & 2,0 \\
\hline Luzern & 122,2 & 135,0 & 1,0 & 3,0 \\
\hline davon: Luzern & 86,0 & 102,0 & & \\
\hline
\end{tabular}


Hilfe für die Transitionsländer (Hilfe für den Osten und die fortgeschritt. Entwicklungsländer)

\begin{tabular}{|c|c|c|c|c|}
\hline & 2000 & 2001 & 2000 & 2001 \\
\hline Neuenburg & 130,3 & 129,0 & 3,0 & 3,0 \\
\hline davon: Ville de Neuchâtel & 77,5 & 73,0 & & \\
\hline Obwalden & 0,3 & 0,0 & & \\
\hline Schaffhausen & 51,7 & 65,0 & & 5,0 \\
\hline davon: Schaffhausen & 48,0 & 57,0 & & \\
\hline Solothurn & 64,1 & 85,0 & 5,0 & 16,0 \\
\hline St. Gallen & 204,3 & 255,0 & 16,5 & 26,0 \\
\hline davon: St.-Gallen & 136,0 & 180,0 & & \\
\hline Jona & 50,0 & 47,0 & & \\
\hline Thurgau & 74,6 & 99,0 & & \\
\hline davon: Frauenfeld & 69,5 & 79,0 & & \\
\hline Tessin & 52,6 & 88,0 & & \\
\hline davon: Lugano & 23,0 & 54,0 & & \\
\hline Uri & 2,5 & 2,0 & - & - \\
\hline Wallis & 107,3 & 51,0 & 10,5 & 20,0 \\
\hline Waadt & 244,3 & 274,0 & 36,9 & 18,0 \\
\hline davon: Lausanne & 146,7 & 160,0 & & \\
\hline Pully & & 43,0 & 57,0 & \\
\hline Zug & 422,0 & 438,0 & 11,5 & 7,0 \\
\hline davon: Zug & 277,0 & 278,0 & & \\
\hline Baar & 140,0 & 150,0 & & \\
\hline Zürich & 1667,3 & 2147,0 & 212,2 & 204,0 \\
\hline davon: Zürich & 500,0 & 800,0 & & \\
\hline Illnau-Effretikon & 210,0 & 178,0 & & \\
\hline Winterthur & 109,0 & 150,0 & & \\
\hline Maur & 98,0 & 142,0 & & \\
\hline Herrliberg & 51,5 & 132,0 & & \\
\hline Kloten & 120,0 & 120,0 & & \\
\hline Zumikon & 0,0 & 75,0 & & \\
\hline Opfikon & 68,0 & 68,0 & & \\
\hline Uitikon Waldegg & 50,0 & 62,0 & & \\
\hline Meilen & 65,0 & 60,0 & & \\
\hline
\end{tabular}

Quelle: DEZA, Statistischer Dienst. 


\subsection{Private Hilfe der NRO, mit privaten Mitteln finanzierte Projekte (2001)}

\begin{tabular}{|c|c|c|c|c|}
\hline Hauptempfängerländer & \multicolumn{2}{|c|}{ Millionen Franken } & $\%$ & $\sum \%$ \\
\hline 1. Bundesrepublik Jugoslawien & 38,6 & 27,2 & 8,2 & 8,2 \\
\hline 2. Indien & 16,8 & 21,6 & 6,5 & 14,8 \\
\hline 3. Kambodscha & 2,6 & 20,0 & 6,0 & 20,8 \\
\hline 4. Mosambik & 9,0 & 11,0 & 3,3 & 24,1 \\
\hline 5. Sudan & 9,9 & 10,7 & 3,2 & 27,3 \\
\hline 6. Brasilien & 7,8 & 8,5 & 2,6 & 29,9 \\
\hline 7. Honduras & 5,6 & 7,0 & 2,1 & 32,0 \\
\hline 8. Kolumbien & 5,7 & 6,9 & 2,1 & 34,1 \\
\hline 9. $\quad$ Tansania & 4,3 & 6,7 & 2,0 & 36,2 \\
\hline 10. $\quad$ Nicaragua & 6,6 & 5,9 & 1,8 & 37,9 \\
\hline 11. Äthiopien & 6,5 & 5,9 & 1,8 & 39,7 \\
\hline 12. Albanien & 2,4 & 5,8 & 1,8 & 41,5 \\
\hline 13. El Salvador & 2,4 & 5,7 & 1,7 & 43,2 \\
\hline 14. Bolivien & 4,9 & 5,6 & 1,7 & 44,9 \\
\hline 15. $\quad$ Peru & 3,1 & 5,5 & 1,7 & 46,5 \\
\hline 16. Philippinen & 5,5 & 5,2 & 1,6 & 48,1 \\
\hline 17. Afghanistan & 3,3 & 5,1 & 1,5 & 49,6 \\
\hline 18. $\quad$ Türkei & 5,3 & 5,0 & 1,5 & 51,2 \\
\hline 19. Bosnien-Herzegowina & 4,3 & 4,8 & 1,5 & 52,6 \\
\hline 20. Demokratische Republik Kongo & 3,8 & 4,5 & 1,3 & 54,0 \\
\hline Andere Länder & 136,8 & 152,2 & 46,0 & 100,0 \\
\hline $\begin{array}{l}\text { Mit privaten Mitteln finanzierte Projekte, } \\
\text { Gesamtbetrag }\end{array}$ & 285,0 & 330,6 & 100,0 & \\
\hline
\end{tabular}

Quelle: DEZA, Statistischer Dienst. 